\title{
LEVELS OF DIVISION ALGEBRAS
}

\author{
by DAVID B. LEEP
}

(Received 7 June, 1989)

Introduction. In [7] the level, sublevel, and product level of finite dimensional central division algebras $D$ over a field $F$ were calculated when $F$ is a local or global field. In Theorem 1.4 of this paper we calculate the same quantities if all finite extensions $K$ of $F$ satisfy $\tilde{u}(K) \leq 2$, where $\tilde{u}$ is the Hasse number of a field as defined in [2]. This occurs, for example, if $F$ is an algebraic extension of the function field $R(x)$ where $R$ is a real closed field or hereditarily Euclidean field (see [4]).

We recall the main definitions here. The level of $D, s(D)$, is the least integer $s$ such that -1 is a sum of $s$ squares in $D$. The sublevel of $D, s(D)$, is the least integer $s$ such that 0 is a sum of $\underline{s}+1$ nonzero squares in $D$. The product level of $D, s_{\pi}(D)$, is the least integer $s_{\pi}$ such that -1 is a sum of $s_{\pi}$ elements which are products of squares in $D$. In each case, $s, \underline{s}$, or $s_{\pi}$ is set equal to $\infty$ if no such representation exists. Clearly $s_{\pi}(D) \leq \underline{s}(D) \leq s(D)$ and if $D$ is a field then all three quantities agree with the usual level of a field.

Section 1 deals with those properties of formally real fields that are useful in calculating levels of division algebras. In Sections 2 and 3 we restrict attention to the case of quaternion division algebras. Additional background to the problem of calculating levels of division algebras may be found in the introduction to [7]. The main references for Sections 2 and 3 are $[\mathbf{9 , 1 0 ]}$.

We use standard terminology from the theory of quadratic forms and ordered fields as found in [6] and [11]. Let $F^{\times}$denote the nonzero elements of $F$. We shall assume throughout that char $F \neq 2$. We let $D_{F}(q)$ denote the nonzero elements of $F$ represented by a quadratic form $q$ over $F$. The topological space of orderings of a field $F$ is denoted $X_{F}$. Basic properties of $X_{F}$ and basic results on SAP fields can be found in [3] and [11].

1. Levels and sublevels of division algebras over formally real fields. In this section we shall assume $-1 \notin F^{2}$, since otherwise $s_{\pi}(D)=s(D)=s(D)=1$ for any division algebra $D$. We recall from [7] that for a cyclic extension $K / F$ of odd degree, $t(K / F)$ is the least integer $t$ for which there exist $a_{1}, \ldots, a_{t} \in K$ such that $N_{K / F}\left(a_{i}\right)=1,1 \leq i \leq t$, and $-1 \in D_{K}\left(\left\langle a_{1}, \ldots, a_{t}\right\rangle\right)$. We will use the following two results from [7].

(1) [7, Proposition 2.4] If $K / F$ is a cyclic extension of odd degree $n>1$, then $t(K / F) \leq n-1$.

(2) [7, Proposition 2.5,2.6] Let $D$ be a division algebra of odd degree over its center $F$. Then $2 \leq \underline{s}(D)$ and $\min \{3, s(F)\} \leq s(D)$. If, in addition, $D$ is a cyclic division algebra and $K$ is a maximal subfield cyclic over $F$, then $\underline{s}(D) \leq t(K / F)$ and $s(D) \leq t(K / F)+1$.

Let $K / F$ be a cyclic extension of degree $n>1$ and let $\sigma$ be an automorphism that generates $\operatorname{Gal}(K / F)$. If $P$ is an ordering on $K$, then $P, \sigma(P), \ldots, \sigma^{n-1}(P)$ are distinct orderings of $K$ and if $a \in K^{\times}$, then $\sigma(a) \in P$ if and only if $a \in \sigma^{-1}(P)$.

Let $\epsilon_{K / F}: X_{K} \rightarrow X_{F}$ be the continuous map defined by restricting an ordering on $K$ to $F$. Then there exists a clopen set $Y$ in $X_{K}$ such that $\left.\epsilon_{K / F}\right|_{Y}$ is a homeomorphism onto $\epsilon_{K / F}\left(X_{K}\right)$. (See [1, p. 139] or [8, Theorem 1.10].)

We claim $X_{K}=\bigcup_{i=0}^{n-1} \sigma^{i}(Y)$ is a disjoint union of clopen sets. Clearly each $\sigma^{i}(Y)$ is 
clopen. If $P \in \sigma^{i}(Y) \cap \sigma^{j}(Y)$ then $P=\sigma^{i}\left(P^{\prime}\right)=\sigma^{j}\left(P^{\prime \prime}\right), P^{\prime}, P^{\prime \prime} \in Y$. It follows that $\epsilon_{K / F}\left(\sigma^{-i}(P)\right)=\epsilon_{K / F}\left(\sigma^{-j}(P)\right)$ and this implies $\sigma^{-i}(P)=\sigma^{-j}(P)$ since each lies in $Y$. Therefore $i=j$. Finally, if $P \in X_{K}$, then $\epsilon_{K / F}(P)=\epsilon_{K / F}\left(P^{\prime}\right)$ for some $P^{\prime} \in Y$. It follows that $P=\sigma^{i}\left(P^{\prime}\right)$ for some $i$, and $P \in \bigcup_{i=0}^{n-1} \sigma^{i}(Y)$.

1.1. LEMMA. Using the notation above, assume also that $K$ is a SAP field. Then there exist $\alpha, \beta \in K^{\times}$such that $\left\langle 1, \frac{\alpha}{\sigma(\alpha)}, \frac{\beta}{\sigma(\beta)}\right\rangle$ is totally indefinite over $K$.

Proof. If $n$ is even, the lemma is trivial since $N_{K / F}(-1)=1$ implies $-1=\frac{\alpha}{\sigma(\alpha)}$ for some $\alpha \in K^{\times}$by Hilbert's Satz 90. Now assume $n$ is odd. Let $D_{1}=\bigcup_{i=0}^{(n-1) / 2} \sigma^{2 i}(Y)$ and let $D_{2}=\bigcup_{i=0}^{(n-1) / 2} \sigma^{2 i+1}(Y)$. Note that $X_{K}=D_{1} \cup D_{2}, D_{1} \cap D_{2}=Y$ and $D_{1}, D_{2}$ are clopen sets. Using the SAP property of $K$, choose $\alpha, \beta \in K^{\times}$such that

$$
\begin{array}{llllll}
\alpha>_{P} 0 & \text { if } & P \in D_{1} & \beta>_{P} 0 & \text { if } & P \in D_{2}, \\
\alpha<{ }_{P} 0 & \text { if } & P \notin D_{1} & \beta<_{P} 0 & \text { if } & P \notin D_{2} .
\end{array}
$$

Then $\frac{\alpha}{\sigma(\alpha)}<{ }_{P} 0$ if $P \in \bigcup_{i=1}^{n-1} \sigma^{i}(Y)$ and $\frac{\beta}{\sigma(\beta)}<{ }_{P} 0$ if $P \in \bigcup_{i=2}^{n} \sigma^{i}(Y)$. Therefore $\left\langle 1, \frac{\alpha}{\sigma(\alpha)}, \frac{\beta}{\sigma(\beta)}\right\rangle$ is totally indefinite over $K$.

1.2. Proposition. Suppose $K / F$ is a cyclic extension of odd degree $n>1$. If $K$ is a $S A P$ field and $K$ satisfies property $A_{m}, m \geq 2$, (every torsion $m$-fold Pfister form defined over $K$ is hyperbolic), then $t(K / F) \leq \min \left\{n-1,2^{m-1}\right\}$.

Proof. In general $t(K / F) \leq n-1$ [7, Proposition 2.4]. Since $K$ is a SAP field we may choose $\alpha, \beta \in K^{\times}$as in Proposition 1.1. Then $q=\left\langle 1, \frac{\alpha}{\sigma(\alpha)}, \frac{\beta}{\sigma(\beta)}\right\rangle$ is a totally indefinite quadratic form defined over $K$ and $\tau=q \perp\left\langle\frac{\alpha \beta}{\sigma(\alpha \beta)}\right\rangle$ is a torsion 2-fold Pfister form over $K$. Therefore $2^{m-2} \tau$ is hyperbolic over $K$, since $K$ satisfies $A_{m}$, and it follows that the subform $\langle 1\rangle \perp 2^{m-3} \tau$ is isotropic over $K$ if $m \geq 3$ and $q$ is isotropic over $K$ if $m=2$. This implies $-1 \in D_{K}\left(2^{m-3} \tau\right)$ if $m \geq 3$ and $-1 \in D_{K}\left(\left\langle\frac{\alpha}{\sigma(\alpha)}, \frac{\beta}{\sigma(\beta)}\right\rangle\right)$ if $m=2$. In each case $t(K / F) \leq 2^{m-1}$.

1.3. Corollary. Suppose $K / F$ is a cyclic extension of odd degree $n>1$. If $\tilde{u}(K)<2^{m}, m \geq 2$, then $t(K / F) \leq \min \left\{n-1,2^{m-1}\right\}$.

Proof. This follows from Proposition 1.2 since $K$ satisfies property $A_{m}, m \geq 2$. Note that $K$ is a SAP field by [4, Theorems B, C]. 
1.4. TheоRem. Let $D \neq F$ be a finite-dimensional crossed product division algebra over a field $F$. Suppose $K$ is a maximal subfield of $D, K / F$ Galois, and $\tilde{u}(K) \leq 2$. Then

(1) $s(D)=\underline{s}(D)=s_{\pi}(D)=1$ if $\operatorname{deg} D$ is even,

(2) $s(D)=\min \{3, s(F)\}$ if $\operatorname{deg} D$ is odd,

(3) $s(D)=s_{\pi}(D)=2$ if $\operatorname{deg} D$ is odd.

(We are still assuming $-1 \notin F^{2}$.)

Proof. First assume $\operatorname{deg} D$ is even. Then $[K: F]$ is even and Galois theory implies there exists a subfield $L$ with $[K: L]=2$ and $\tilde{u}(L) \leq 2$ by [5, Proposition 3.3]. The centralizer of $L$ in $D$ is then a quaternion algebra $(a, b)_{L}$ with $a, b \in L$. The quadratic form $q=\langle 1, a, b,-a b\rangle$ is isotropic over $L$ since $q$ is totally indefinite over $L$ and $\tilde{u}(L) \leq 2$. Therefore $-1=a \alpha^{2}+b \beta^{2}-a b \gamma^{2}=(\alpha i+\beta j+\gamma k)^{2}$ for some $\alpha, \beta, \gamma \in L$ and where $i, j, k=i j$ is the standard basis of $(a, b)_{L}$. This shows $s(D)=1$ and hence $\underline{s}(D)=s_{\pi}(D)=1$.

Now assume deg $D$ is odd. Then $\operatorname{Gal}(K / F)$ has odd order and $K$ contains a subfield $L$ corresponding to a subgroup of prime order. Thus $K / L$ is a cyclic extension of odd degree $>1$. The centralizer of $L$ in $D$ is a cyclic algebra $E$ of odd degree over its center $L$. From [7, Proposition 2.6] we have $2 \leq \underline{s}(D)$ and $\min \{3, s(F)\} \leq s(D)$. From [7, Proposition 2.5] and Corollary 1.3 we have $\underline{s}(D) \leq \underline{s}(E) \leq t(K / L) \leq 2$ and $s(D) \leq s(E) \leq t(K / L)+1 \leq 3$. Since $s(D) \leq s(F)$ we conclude that $s(D)=\min \{3, s(F)\}$ and $s(D)=2$. We have $2 \leq s_{\pi}(D) \leq \underline{s}(D)$ since deg $D$ is odd [7, Proposition 1.1] and therefore $s_{\pi}(D)=2$.

2. Levels and sublevels of quaternion algebras. Levels and sublevels of quaternion algebras were considered in [9] and [10]. We give several additional results in this section. For convenience we list some of Lewis's results below in Proposition 2.1.

Let $D=\left(\frac{a, b}{F}\right)$ be a quaternion algebra with standard basis $\{1, i, j, k=i j\}$ where $i^{2}=a, j^{2}=b, j i=-i j$. Following the notation in $[9,10]$, let $T_{D}=\langle 1, a, b,-a b\rangle$ and $T_{P}=\langle a, b,-a b\rangle$. We will consider the equation $c=\sum_{\lambda=1}^{n}\left(x_{\lambda}+y_{\lambda} i+z_{\lambda} j+w_{\lambda} k\right)^{2}$ with $c=0$ or -1 . Let $\vec{x}=\left(x_{1}, \ldots, x_{n}\right), \ldots, \vec{w}=\left(w_{1}, \ldots, w_{n}\right)$. Then this equation is equivalent to $c=\sum x_{\lambda}^{2}+a \sum y_{\lambda}^{2}+b \sum z_{\lambda}^{2}-a b \sum w_{\lambda}^{2}$ and $\vec{x} \cdot \vec{y}=\vec{x} \cdot \vec{z}=\vec{x} \cdot \vec{w}=0$.

Note that $s_{\pi}(D)=1$ for all quaternion algebras $D$ since $i^{2} j^{2}(i j)^{-2}=-1$. Also note that if $D$ is a split algebra, then we may assume $a=1$. In this case $T_{P}$ is isotropic and the next result shows $s(D)=1$.

2.1. Proposition. (1) [9, Lemmas 2, 4] If $\langle 1\rangle \perp n T_{P}$ is isotropic over $F$, then $s(D) \leq n$. The converse holds if $n=2^{k}-1, k \geq 2$. If $k=1$, then $s(D)=1$ if and only if either $T_{D}$ is isotropic or $-1 \in F^{2}$.

(2) [10, Proposition 2] If either $\langle 1\rangle \perp n T_{P}$ or $(n+1) T_{P}$ is isotropic over $F$, then $\leqq(D) \leq n$. The converse holds if $n=2^{k}-1, k \geq 1$.

Lewis proved the "only if" direction of the following result in [9, Lemma 3].

2.2. THEOREM. For $k \geq 0, s(D) \leq 2^{k}$ if and only if either (1) or (2) below holds.

(1) $\left(2^{k}+1\right)\langle 1\rangle \perp\left(2^{k}-1\right) T_{P}$ is isotropic over $F$.

(2) $\langle 1\rangle \perp 2^{k} T_{P}$ is isotropic over $F$. 
Proof. We prove the "if" direction here. If (2) holds then $s(D) \leqq 2^{k}$ by Proposition 2.1 (1). Now assume (1) holds. Then there exists $-A \in D_{F}\left(\langle 1\rangle \perp\left(2^{k}-1\right) T_{P}\right)$ for some nonzero $A \in D_{F}\left(2^{k}\langle 1\rangle\right)$. Hence for some $\alpha \in F$ and $B, C, D \in D_{F}\left(\left(2^{k}-1\right)\langle 1\rangle\right) \cup\{0\}$ we have

$$
-A=\alpha^{2}+a B+b C-a b D, \quad \text { i.e., } \quad-1=\frac{1}{A^{2}}\left(\alpha^{2} A+a A B+b A C-a b A D\right) .
$$

Let $A=\sum_{\lambda=1}^{2^{k}} x_{\lambda}^{2}$. We show now there exist $y_{\lambda} \in F$ such that $\sum_{\lambda=1}^{2^{k}} y_{\lambda}^{2}=A B$ and $\vec{x} \cdot \vec{y}=0$. If $B=0$, let each $y_{\lambda}=0$. If $B \neq 0$, then $\langle A, A B\rangle \cong A\langle 1, B\rangle$ is a subform of $A \cdot 2^{k}\langle 1\rangle \cong$ $2^{k}\langle 1\rangle$ since $B \in D_{F}\left(\left(2^{k}-1\right)\langle 1\rangle\right)$ and $A \in D_{F}\left(2^{k}\langle 1\rangle\right)$. Therefore such a $\vec{y}$ exists. Similarly $\vec{z}, \vec{w}$ exist such that $\sum_{\lambda=1}^{2^{k}} z_{\lambda}^{2}=A C, \sum_{\lambda=1}^{2^{k}} w_{\lambda}^{2}=A D$ and $\vec{x} \cdot \vec{z}=\vec{x} \cdot \vec{w}=0$. It follows that

$$
\sum_{\lambda=1}^{2^{k}}\left(\frac{\alpha x_{\lambda}}{A}+\frac{y_{\lambda}}{A} i+\frac{z_{\lambda}}{A} j+\frac{w_{\lambda}}{A} k\right)^{2}=\frac{1}{A^{2}}\left(\alpha^{2} A+a A B+b A C-a b A D\right)=-1 .
$$

Therefore $s(D) \leq 2^{k}$.

2.3. Lemma. Suppose $2^{k} T_{P}$ is isotropic, $k \geq 0$. Then $\left(1+\left[\frac{2}{3} .2^{k}\right]\right) T_{P}$ is isotropic. ([ ] is the greatest integer function.)

Proof. If $2^{k} T_{P}$ is isotropic then $2^{k}\langle-a,-b, a b\rangle$ is isotropic and $2^{k}\langle\langle-a,-b\rangle\rangle$ is hyperbolic. After multiplying by -1 we see that any subform of $2^{k}\langle-1, a, b,-a b\rangle$ of dimension greater than $2.2^{k}$ is isotropic. The conclusion follows since $3\left(1+\left[\frac{2}{3} \cdot 2^{k}\right]\right)>$ $3\left(\frac{2}{3} .2^{k}\right)=2.2^{k}$.

2.4. Proposition. If $k \geq 2$, then $\underline{s}(D) \leq 2^{k}-1$ implies $s(D) \leq 2^{k}-1$.

Proof. If $\underline{s}(D) \leq 2^{k}-1$ then by Proposition $2.1(2)$, either $\langle 1\rangle \perp\left(2^{k}-1\right) T_{P}$ or $2^{k} T_{P}$ is isotropic. If $\langle 1\rangle \perp\left(2^{k}-1\right) T_{P}$ is isotropic, then $s(D) \leq 2^{k}-1$ by Proposition $2.1(1)$. If $2^{k} T_{P}$ is isotropic, then $\left(1+\left[\frac{2}{3} .2^{k}\right]\right) T_{P}$ is isotropic by Lemma 2.3. Then Proposition 2.1(1) implies $s(D) \leq 1+\left[\frac{2}{3} .2^{k}\right] \leq 2^{k}-1$ since $k \geq 2$.

Remark. This result is a slight improvement of [10, Proposition 4]. $2 s(D)$.

2.5. TheOREM. (1) If $\underline{s}(D)=1$, then $s(D) \leq 2$ and if $2 \leq \underline{s}(D)<\infty$, then $s(D)<$

(2) If $\underline{s}(D)=2^{k}-1, k \geq 2$, then $s(D)=2^{k}-1$.

(3) If $s(D)=2^{k}, k \geq 2$, then $\underline{s}(D)=2^{k}$.

(4) If $s(D)=2^{k}+1, k \geq 1$, then $\underline{s}(D)=2^{k}$ or $2^{k}+1$.

Proof. (1) If $s(D)=1$, then $s(D) \leqq 2$ by Proposition 2.1(2) and Theorem 2.2. If $2 \leq \underline{s}(D)<\infty$, then $s(D)<2 s(D)$ by Proposition 2.4 .

If $k \geq 2$, then (2), (3), (4) all follow from Proposition 2.4 and the estimate $\underline{s}(D) \leq s(D)$. If $k=1$ in $(4)$ and $s(D)=3$, then $\underline{s}(D) \leq 3$. Since $s(D)=1$ implies $s(D) \leq 2$ by (1), it follows that $s(D)=3$ implies $\underline{s}(D)=2$ or 3 .

The next section deals with examples where the ordered pair $(\underline{s}(D), s(D))$ has been computed. 
3. Examples of levels and sublevels of quaternion division algebras. We continue the notation of Section 2 . If $F$ is a local or global field then $(s(D), s(D))$ was calculated in [7] and we had $s(D) \leq s(D) \leq 2$ in all cases. If $\tilde{u}(F) \leq 2$, then $(s(D), s(D))$ was calculated in Theorem 1.4 and we found $(s(D), s(D))=(1,1)$. (The maximal subfield $K$ satisfies $\tilde{u}(K) \leq 2$ by [5, Proposition 3.3].) In [12], $s(D)$ was calculated if $D=\left(\frac{a, t}{F((t))}\right)$ where $a$ is a nonsquare in $F^{\times}$and $F((t))$ is the field of formal power series over $F$. We review this result below and also calculate $\underline{s}(D)$.

If $a \in F^{\times}$, let $g(a)$ be the least integer such that $g(a)\langle 1,-a\rangle$ is isotropic over $F$ and set $g(a)=\infty$ if no such integer exists.

3.1. THEоREM [12]. (1) Let $D=\left(\frac{a, t}{F((t))}\right)$ where a is a nonsquare in $F^{\times}$. Then $s(D)=$ $\min \{g(a), s(F(\sqrt{a}))\}$.

(2) Let $F=\mathbb{R}\left(x_{1}, \ldots, x_{n}\right)$, the rational function field in $n$ variables over the real numbers, and assume $n \geq 2$. Let $a=\sum_{i=1}^{n} x_{i}^{2}$ and let $D=\left(\frac{a, t}{F((t))}\right), D^{\prime}=\left(\frac{-a, t}{F((t))}\right)$. Then $s(D)=2^{k}+1$ if $2^{k}<n \leq 2^{k+1}$ and $s\left(D^{\prime}\right)=2^{k}$ if $2^{k} \leq n<2^{k+1}$.

It was shown in [12] that $g(a)$ always has the form $2^{k}+1$ if $g(a)$ is finite. Since the level of a field is always a power of 2 if finite, we see that for $D$ as in Theorem 3.1(1), $s(D)=2^{k}$ or $2^{k}+1$ if $s(D)<\infty$.

3.2. THEOREM. (1) Let $D$ be as in Theorem 3.1(1). Then

$$
\underline{s}(D)=\min \{g(a)-1, s(F(\sqrt{a}))\} \text {. }
$$

(2) Let $D, D^{\prime}$ be as in Theorem 3.1(2). Then $s(D)=2^{k}$ if $2^{k}<n \leq 2^{k+1}$ and $\underline{s}\left(D^{\prime}\right)=2^{k}$ if $2^{k} \leq n<2^{k+1}$.

We omit the proof of Theorem 3.2 since it is so similar to the proof in [12]. Note that the result for $D^{\prime}$ in (2) with $k \geq 2$ follows from Theorems 2.5(3) and 3.1(2).

The examples mentioned here show that the following values of the ordered pair $(s(D), s(D))$ can occur when $D$ is a quaternion algebra:

$$
(\underline{s}(D), s(D))=\left(2^{k}, 2^{k}\right) \quad \text { or }\left(2^{k}, 2^{k}+1\right), \quad k \geq 0 .
$$

The main questions to consider on levels and sublevels of quaternion algebras $D$ are the following.

(1) Is it always true that $\underline{s}(D)=2^{k}$ ?

(2) Is it always true that $s(D)=2^{k}$ or $2^{k}+1$ ?

(3) Is it always true that $s(D) \leq \underline{s}(D)+1$ ?

Added in proof. Krüskemper and Wadsworth have constructed a quaternion division algebra $D$ with $\underline{s}(D)=3$. By Theorem 2.5(2), it follows that $s(D)=3$. Thus the answer to question (1) is no.

\section{REFERENCES}

1. R. Bos, Quadratic forms, orderings and abstract Witt rings, Dissertation (Rijksuniversiteit te Utrecht, 1984). 
2. R. Elman, Quadratic forms and the $u$-invariant III, in G. Orzech, editor, Quadratic forms conference, 1976, Queen's Papers in Pure and Applied Mathematics 46 (1977), 422-444.

3. R. Elman and T. Y. Lam, Quadratic forms over formally real fields and Pythagorean fields, Amer. J. Math. 94 (1972), 1155-1194.

4. R. Elman, T. Y. Lam and A. Prestel, On some Hasse principles over formally real fields, Math. Z. 134 (1973), 291-301.

5. R. Elman, T. Y. Lam and A. Wadsworth, Quadratic forms under multiquadratic extensions, Nederl. Akad. Wetensch. Indag. Math. 42 (1980), 131-145.

6. T. Y. Lam, The algebraic theory of quadratic forms (Benjamin, 1973).

7. D. B. Leep, J.-P. Tignol and N. Vast, The level of division algebras over local and global fields. J. Number. Theory 33 (1989), 53-70.

8. D. B. Leep and A. R. Wadsworth, The transfer ideal of quadratic forms and a Hasse norm theorem mod squares, Trans. Amer. Math. Soc. 315 (1989), 415-431. 787-792.

9. D. W. Lewis, Levels of quaternion algebras, Rocky Mountain J. Math., 19 (1989),

10. D. W. Lewis, Levels and sublevels of division algebras, Proc. Roy. Irish Acad. Sect. A 87 (1987), 103-106.

11. W. Scharlau, Quadratic forms and hermitian forms (Springer, 1985).

12. J.-P. Tignol and N. Vast, Représentation de -1 comme somme de carrés dans certaines algèbres de quaternions, C. R. Acad. Sci. Paris Sér. I Math. 305 (1987), 583-586.

Department of Mathematics

UNIVERSITY OF KENTUCKY

LEXINGTON KY 40506

USA 